\title{
Temporal trends in neonatal outcomes following iatrogenic preterm delivery
}

Sarka Lisonkova ${ }^{{ }^{*}}$, Jennifer A Hutcheon ${ }^{1}$ and KS Joseph ${ }^{1,2}$

\begin{abstract}
Background: Preterm birth rates have increased substantially in the recent years mostly due to obstetric intervention. We studied the effects of increasing iatrogenic preterm birth on temporal trends in perinatal mortality and serious neonatal morbidity in the United States.

Methods: We used data on singleton and twin births in the United States, 1995-2005 ( $n=36,399,333$ ), to examine trends in stillbirths, neonatal deaths, and serious neonatal morbidity (5-minute Apgar $\leq 3$, assisted ventilation $\geq 30$ min and neonatal seizures). Preterm birth subtypes were identified using an algorithm that categorized live births $<37$ weeks into iatrogenic preterm births, births following premature rupture of membranes and spontaneous preterm births. Temporal changes were quantified using odds ratios (OR) and 95\% confidence intervals (Cl).

Results: Among singletons, preterm birth increased from 7.3 to 8.8 per 100 live births from 1995 to 2005, while iatrogenic preterm birth increased from 2.2 to 3.7 per 100 live births. Stillbirth rates declined from 3.4 to 3.0 per 1,000 total births from 1995-96 to 2004-05, and neonatal mortality rates declined from 2.4 to 2.1 per 1,000 live births. Temporal declines in neonatal mortality/morbidity were most pronounced at 34-36 weeks gestation and larger among iatrogenic preterm births $(\mathrm{OR}=0.75, \mathrm{Cl} 0.73-0.77)$ than among spontaneous preterm births $(\mathrm{OR}=$ $0.82, \mathrm{Cl} 0.80-0.84) ; \mathrm{P}<0.001$. Similar patterns were observed among twins, with some notable differences.
\end{abstract}

Conclusion: Increases in iatrogenic preterm birth have been accompanied by declines in perinatal mortality. The temporal decline in neonatal mortality/serious neonatal morbidity has been larger among iatrogenic preterm births as compared with spontaneous preterm births.

\section{Background}

Preterm birth is the leading cause of neonatal mortality and morbidity and preterm infants are more likely to experience neurodevelopmental delay and childhood disability. Thus, the recent increase in preterm birth that has been observed in many industrialized countries is a cause for concern. For instance, in the United States, the rate of preterm birth increased by $20 \%$ from $10.6 \%$ in 1990 to $12.7 \%$ in 2007 [1,2], whereas in Canada the rate of preterm birth increased by $18 \%$ from $6.6 \%$ in 1990 to $7.8 \%$ in 2007 [3,4].

Preterm birth can result from many maternal and fetal causes. Three major clinical subtypes of preterm birth can be identified, namely, iatrogenic (medically

\footnotetext{
* Correspondence: slisonkova@cfri.ca

'Department of Obstetrics \& Gynaecology, University of British Columbia and the Women's Hospital and Health Centre of British Columbia, Vancouver,

Canada

Full list of author information is available at the end of the article
}

indicated) preterm birth, spontaneous preterm birth, and preterm birth following premature rupture of membranes. In North America, the recent increases in preterm birth occurred predominantly due to increases in iatrogenic preterm birth at late preterm gestation (34-36 weeks) $[5,6]$. Other factors in the increase in preterm birth have included changes in maternal characteristics (such as increases in older maternal age) [7-10] and in the frequency of multiple births $[11,12]$.

Infants born following medically indicated preterm birth are at a two-fold higher risk of neonatal mortality as compared with infants born following spontaneous preterm birth [13-15]. If the risks of adverse birth outcomes among the different preterm birth subtypes have remained unchanged, a temporal increase in preterm neonatal mortality could be expected given the recent increase in iatrogenic preterm births. On the other hand, the recent increases in medically indicated preterm birth have followed improvements in fetal

\section{Biomed Central}


surveillance, obstetric care and neonatal care. This could have resulted in differential reductions in neonatal mortality and serious neonatal morbidity among infants born following iatrogenic preterm birth (as compared with those born following spontaneous preterm birth).

Our goal was to estimate temporal trends in iatrogenic and spontaneous preterm birth and to quantify trends in stillbirth, neonatal mortality and serious neonatal morbidity among the different preterm birth subtypes. We hypothesized that, over the past decade, there have been larger declines in neonatal mortality and serious neonatal morbidity among preterm infants born following obstetric intervention, as compared with infants born following spontaneous preterm labour.

\section{Methods}

We used population-based data on singleton and twin births in the United States, 1995-2005, from the National Centre for Health Statistics (NCHS). Information in the NCHS period linked birth and death files, and fetal death files was abstracted from birth certificates of liveborn infants and from fetal and infant death certificates [16,17]. These data files provided gestational age estimates based on menstrual dates and also the clinical estimate of gestation [18]. The menstrual estimate of gestational age was estimated by the NCHS based on the date of the last normal menstrual period, with the day imputed if missing. The clinical estimate of gestation was that provided by the health care provider, without specification of the source (i.e., whether based on clinical examination, ultrasound, etc). For this study we used the latter, more accurate clinical estimate of gestation at birth [19-21]. We excluded infants born before 24 weeks of gestation, and those weighing less than 500 grams in order to avoid potential bias due to variable birth registration at the borderline of viability [22-24], as attitudes toward such registration may have changed over time. We further excluded infants with a missing clinical estimate of gestational age, a gestational age $>45$ weeks, or missing data on birth weight or mode of delivery. We excluded an additional $10.9 \%$ of births in 2004-05 due to missing data on rupture of membranes; these missing data was related to the introduction of the new birth certificate by some states in 200405 . Sensitivity analyses were carried out to account for this limitation (see below).

Preterm birth was defined as live birth before 37 completed weeks of gestation, and classified into 3 subtypes using a previously published algorithm $[6,15]$. Since the NCHS files do not contain direct information on preterm birth subtypes, this algorithm used information on premature rupture of membranes, labour induction, etc, to assign the preterm birth subtype in the following sequence [6,15]: 1) preterm birth following premature rupture of membranes for over 12 hours (PROM); 2) iatrogenic preterm birth (preterm birth following labour induction or caesarean delivery without PROM or conditions indicating prior onset of labour); 3) spontaneous preterm birth (all other births). In the absence of labour induction, preterm birth following caesarean delivery that occurred after the onset of labour (indicated by mention of complications such as precipitous labour, prolonged labour, cephalopelvic disproportion or dysfunctional labour) was assigned to the spontaneous preterm birth category.

Neonatal death was defined as death of an infant that occurred within the first 28 days after birth and serious neonatal morbidity was defined as any of the following conditions: a 5-minute Apgar score $\leq 3$, assisted ventilation $\geq 30$ minutes and neonatal seizures. A composite measure including neonatal death or any of the serious neonatal morbidity listed above was used to estimate the overall rate of adverse neonatal outcomes.

We examined potential differences in maternal characteristics between 1995-96 and 2004-05, with respect to age, race (non-hispanic white, non-hispanic black, hispanic, other), marital status (married or common-law vs. other), education (<12 years vs. 12 years or more), smoking during pregnancy (yes/no) and prior live births (yes/no). We also examined infants' gender, gestational age distributions among stillbirths and neonatal deaths, and the birth prevalence of congenital anomalies.

Temporal trends were quantified by contrasting neonatal mortality and neonatal mortality/serious neonatal morbidity between 1995-96 and 2004-05 using odds ratios and 95\% confidence intervals (CI). Temporal changes were further examined within gestational age categories 24-27, 28-31, 32-33, and 34-36 weeks. Odds ratios were reported separately for singletons and twins. Differences in the magnitude of the temporal decline in neonatal mortality or neonatal mortality/serious neonatal morbidity between subtypes of preterm birth (e.g., between the odds ratios expressing the temporal declines in neonatal mortality among preterm birth following iatrogenic delivery vs. spontaneous preterm birth) were assessed using a test for heterogeneity of the odds ratios [25]. We also carried out supplementary analyses to assess if our results were affected by the exclusion of infants with congenital anomalies since temporal increases in prenatal diagnosis and pregnancy termination may have influenced neonatal mortality trends. Sensitivity analyses were also carried out to examine if the exclusion of states which introduced a new birth certificate form in 2004 or 2005 affected our results. Finally, supplementary analyses were carried out to examine if there were temporal difference in outcomes among subgroups such as older mothers $(\geq 35$ years). Data used in this study were publicly accessible 
from the National Centre for Health Statistics. All analyses were performed using SAS statistical package version 9.1.3 (SAS Institute Inc., Cary, NC).

\section{Results}

Maternal characteristics changed during the study period: women who delivered in 2004-05 were older, more educated and smoked less during pregnancy compared with women who delivered in 1995-96 (Table 1). The proportion of births to mothers of Hispanic origin, unmarried mothers and mothers with no prior live births increased, while the frequency of congenital anomalies decreased. The rates of stillbirth and neonatal mortality decreased in all gestational age categories among both singletons and twins (Table 1). The largest declines in stillbirth and neonatal mortality rates occurred at late preterm and term gestational ages.

The overall rate of preterm birth increased from 8.4 per 100 live births in 1995 to 10.5 per 100 live births in 2005. Late preterm births (34-36 weeks), which increased from 5.3 per 100 live births in 1995 to 6.7 per 100 live births in 2005, were responsible for most of the increase. Preterm birth rates increased from 7.3 in 1995 to 8.8 per 100 live births in 2005 among singletons (odds ratio $=1.22,95 \% \mathrm{CI}: 1.21-1.23$ ) and from 52.3 to 62.0 per 100 live births among twins (odds ratio $=1.49$, 95\%CI:1.46-1.52).

The increase in the singleton preterm birth rate was predominantly due to an increase in iatrogenic preterm birth from 2.2 in 1995 to 3.7 per 100 live births in 2005

Table 1 Maternal and Infant Characteristics Among Singletons and Twins Born at 24 Weeks of Gestation or Later, United States, 1995-96 and 2004-05

\begin{tabular}{|c|c|c|c|c|c|c|}
\hline \multirow[t]{2}{*}{ Maternal characteristics* } & \multicolumn{3}{|c|}{ Singletons } & \multicolumn{3}{|c|}{ Twins } \\
\hline & $1995-96$ & 2004-05 & Odds ratio $(95 \% \mathrm{Cl})$ & $1995-96$ & 2004-05 & Odds ratio $(95 \% \mathrm{Cl})$ \\
\hline No. of women & $6,359,866$ & $6,122,022$ & & 82,639 & 101,572 & \\
\hline Age (years) $<20$ & 13.3 & 10.6 & $0.77(0.77-0.77)$ & 7.24 & 5.14 & $0.70(0.68-0.71)$ \\
\hline $20-24$ & 24.8 & 25.8 & $1.06(1.05-1.06)$ & 19.6 & 18.0 & $0.90(0.89-0.92)$ \\
\hline $25-29$ & 27.5 & 27.4 & $0.99(0.99-1.00)$ & 27.7 & 26.1 & $0.92(0.91-0.92)$ \\
\hline $30-34$ & 23.0 & 22.7 & $0.98(0.98-0.99)$ & 28.9 & 29.1 & $1.01(1.00-1.03)$ \\
\hline $35-39$ & 9.72 & 11.0 & $1.15(1.15-1.15)$ & 14.2 & 16.9 & $1.23(1.20-1.25)$ \\
\hline $40+$ & 1.73 & 2.45 & $1.43(1.42-1.44)$ & 2.44 & 4.73 & $1.98(1.91-2.06)$ \\
\hline Race: Non-Hispanic white & 66.2 & 58.9 & $0.73(0.73-0.73)$ & 69.2 & 65.5 & $0.84(0.83-0.85)$ \\
\hline African American & 16.5 & 15.4 & $0.93(0.92-0.93)$ & 18.1 & 16.8 & $0.91(0.90-0.93)$ \\
\hline Hispanic & 13.3 & 20.2 & $1.64(1.64-1.65)$ & 9.81 & 13.4 & $1.42(1.39-1.45)$ \\
\hline Other & 4.04 & 5.56 & $1.40(1.39-1.41)$ & 2.89 & 4.31 & $1.51(1.46-1.57)$ \\
\hline Maternal education $<12$ years & 20.8 & 20.6 & $0.98(0.98-0.98)$ & 15.3 & 13.6 & $0.84(0.83-0.86)$ \\
\hline Smoking during pregnancy & 13.8 & 11.1 & $0.78(0.78-0.78)$ & 12.4 & 9.00 & $0.70(0.68-0.71)$ \\
\hline Unmarried & 32.4 & 36.8 & $1.22(1.21-1.22)$ & 27.9 & 28.6 & $1.03(1.02-1.05)$ \\
\hline No prior live births & 42.3 & 40.4 & $0.93(0.92-0.93)$ & 21.9 & 22.4 & $1.02(1.01-1.04)$ \\
\hline Infant sex (male) & 51.2 & 51.2 & $1.00(1.00-1.00)$ & 49.9 & 50.2 & $1.01(1.00-1.02)$ \\
\hline Congenital anomalies (yes) & 1.59 & 1.17 & $0.74(0.73-0.74)$ & 2.44 & 1.66 & $0.69(0.66-0.72)$ \\
\hline \multicolumn{7}{|l|}{ Gestational age-specific } \\
\hline Stillbirths: 24-27 wks & 13.4 & 11.6 & $0.85(0.81-0.90)$ & 6.16 & 4.66 & $0.74(0.64-0.87)$ \\
\hline $28-31$ & 7.78 & 6.91 & $0.88(0.84-0.92)$ & 3.10 & 1.89 & $0.60(0.51-0.70)$ \\
\hline $32-33$ & 4.19 & 3.51 & $0.83(0.78-0.88)$ & 1.26 & 0.59 & $0.47(0.37-0.59)$ \\
\hline $34-36$ & 1.21 & 0.95 & $0.78(0.74-0.81)$ & 0.51 & 0.28 & $0.54(0.45-0.63)$ \\
\hline$>=37$ & 0.13 & 0.11 & $0.81(0.79-0.84)$ & 0.24 & 0.15 & $0.61(0.49-0.76)$ \\
\hline All & 0.34 & 0.30 & $0.88(0.86-0.89)$ & 0.80 & 0.51 & $0.64(0.59-0.69)$ \\
\hline Neonatal deaths: 24-27 wks & 18.5 & 16.4 & $0.87(0.83-0.91)$ & 23.0 & 18.5 & $0.76(0.69-0.83)$ \\
\hline $28-31$ & 4.11 & 3.69 & $0.89(0.84-0.95)$ & 3.03 & 2.54 & $0.83(0.72-0.97)$ \\
\hline $32-33$ & 1.89 & 1.63 & $0.88(0.80-0.96)$ & 0.71 & 0.62 & $0.86(0.66-1.12)$ \\
\hline $34-36$ & 0.61 & 0.50 & $0.81(0.77-0.87)$ & 0.39 & 0.25 & $0.65(0.53-0.78)$ \\
\hline$>=37$ & 0.10 & 0.08 & $0.76(0.73-0.79)$ & 0.20 & 0.11 & $0.55(0.43-0.72)$ \\
\hline All & 0.24 & 0.21 & $0.87(0.85-0.89)$ & 1.17 & 1.95 & $0.80(0.75-0.86)$ \\
\hline
\end{tabular}

*Women with missing information excluded: 5-8\% for smoking status and 1-5\% for congenital anomalies. All proportions based on live births except for stillbirth rates which were based on total births.

All differences in maternal/infant characteristics between 1995-96 and 2004-05 were statistically significant $(P<0.05)$ except for the differences in infant sex and the proportion of twin births at 32-33 weeks of gestation. 
(odds ratio $=1.77,95 \% \mathrm{CI}: 1.76-1.79$; Figure 1 ). The rate of spontaneous preterm birth among singletons was 4.1 per 100 live births in 1995 and 4.2 per 100 live births in 2005, while the rate of PROM preterm birth was 1.0 per 100 live births in 1995 and 0.9 per 100 live births in 2005. Among twins, the iatrogenic preterm birth rate increased from 24.9 to 39.8 per 100 live births from 1995 to 2005 (odds ratio $=2.07,95 \%$ CI:2.03-2.11), while the spontaneous preterm birth rate declined from 21.7 to 16.9 per 100 live births over the same period (odds ratio $=0.70,95 \% \mathrm{CI}: 0.69-0.72$; Figure 1 ). Rates of preterm birth following PROM were 5.7 in 1995 and 5.4 per 100 live births in 2005 (Figure 1).
Neonatal mortality rates declined from 2.5 to 1.9 per 100 preterm live births between 1995-96 and 2004-05 among singletons born following iatrogenic preterm delivery and from 1.6 to 1.2 per 100 preterm live births among singletons born spontaneously. The magnitude of the decline in neonatal mortality following iatrogenic preterm birth (odds ratio $=0.75,95 \%$ CI:0.71-0.78) was not significantly greater than the decline following spontaneous preterm birth (odds ratio $=0.78,95 \%$ CI:0.74$0.81 ; P$ value for difference in odds ratios $=0.21$ ). There was no significant change in neonatal mortality rates among live births following preterm rupture of membranes between 1995-96 and 2004-05 (odds ratio $=0.95$,
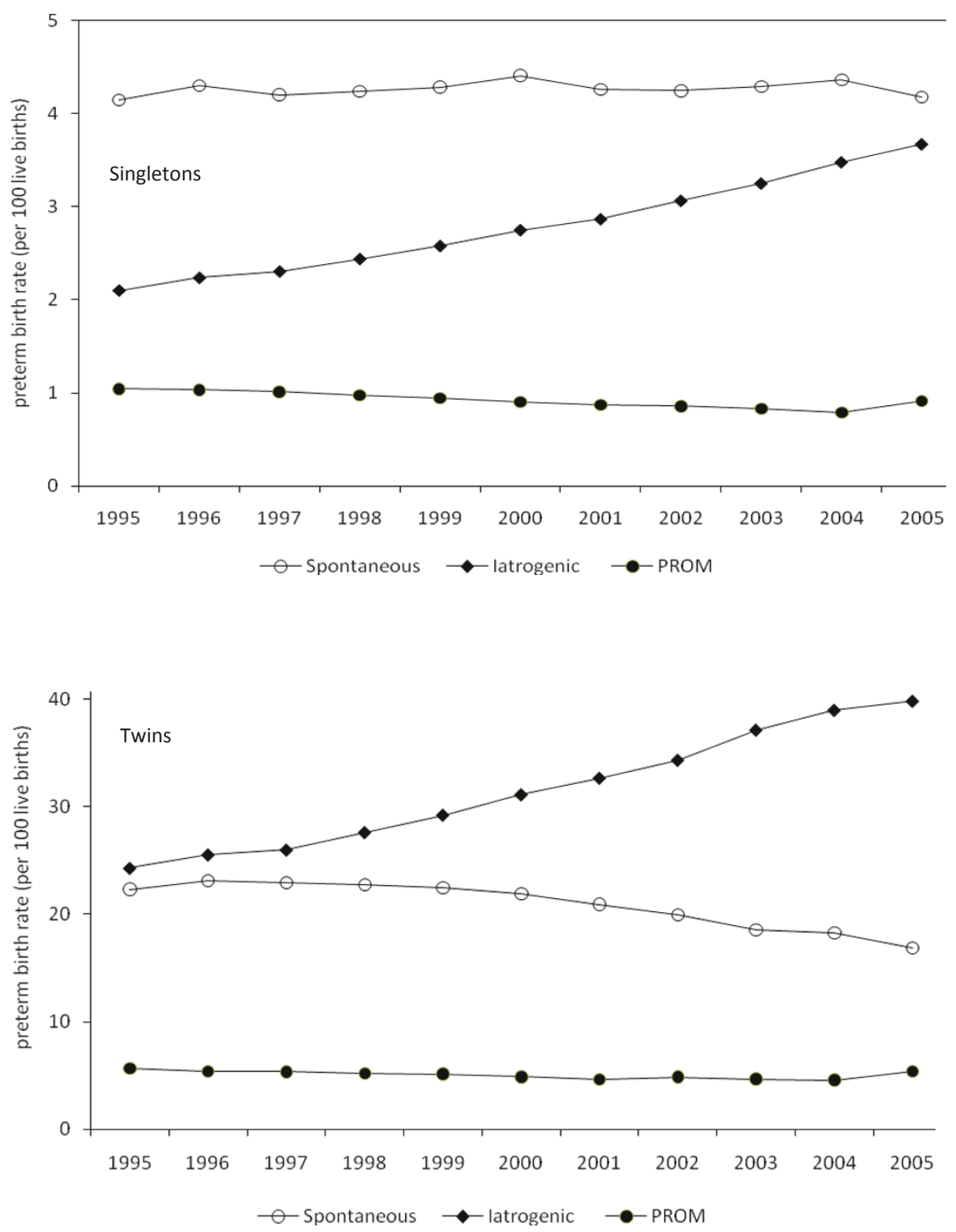

Figure 1 Preterm birth rates per 100 live births among singletons and twins by subtype of preterm birth, namely, spontaneous preterm birth, iatrogenic preterm birth and preterm birth following premature rupture of membranes for over 12 hours (PROM), United States, 1995 to 2005. 
95\%CI:0.88-1.02). The difference in temporal trends in neonatal mortality between infants born after PROM and those born following iatrogenic and spontaneous preterm birth was significant $(P$ value $<0.001$ for both contrasts). In general, larger reductions in neonatal mortality were observed at late preterm gestation (34-36 weeks) compared to earlier gestational ages (24-27, 3831 and 32-33 weeks) (Table 2).

Among twins, neonatal mortality rates between 199596 and 2004-05 declined significantly among all 3 preterm birth subtypes. The magnitude of the decline in neonatal mortality among the 3 preterm birth subtypes was similar $(P>0.05$ for all 3 contrasts). The observed declines in neonatal mortality within each preterm birth subtype were largest among live births at 34-36 weeks gestation (Table 2).

Composite neonatal mortality or serious neonatal morbidity showed a different pattern of change between 1995-96 and 2004-05 (Table 3). The reduction in neonatal mortality/serious neonatal morbidity among singletons was greater among infants born following iatrogenic preterm birth compared with those born following spontaneous preterm birth (odds ratio $=0.75$, 95\%CI:0.73-0.77 vs. 0.82 , 95\%CI:0.80-0.84; $P$ value for difference in odds ratios $<0.001)$. Neonatal mortality/serious neonatal morbidity rates among preterm live births following PROM increased significantly from 1995-96 to 2004-05 and this change was significantly different from

Table 2 Temporal Changes in Neonatal Mortality by Plurality, Gestational Age and Subtype of Preterm Birth, United States, 1995-96 and 2004-05

\begin{tabular}{|c|c|c|c|c|c|c|}
\hline \multirow[b]{2}{*}{$\begin{array}{l}\text { Plurality and gestational age } \\
\text { and preterm birth subtype }\end{array}$} & \multicolumn{2}{|c|}{ 1995-96 } & \multicolumn{2}{|c|}{ 2004-05 } & \multirow[b]{2}{*}{$\begin{array}{l}\text { Odds ratio }(2004- \\
05 \text { vs } 1995-96)\end{array}$} & \multirow[b]{2}{*}{$\begin{array}{c}95 \% \\
\text { confidence } \\
\text { intervals }\end{array}$} \\
\hline & $\begin{array}{l}\text { Number of } \\
\text { live births }\end{array}$ & $\begin{array}{l}\text { Neonatal deaths/ } \\
100 \text { live births }\end{array}$ & $\begin{array}{l}\text { Number of } \\
\text { live births }\end{array}$ & $\begin{array}{l}\text { Neonatal deaths/ } \\
100 \text { live births }\end{array}$ & & \\
\hline \multicolumn{7}{|l|}{ Singletons } \\
\hline 24-27 wks: latrogenic & 7,580 & 17.3 & 10,202 & 16.2 & 0.92 & $0.85-1.00$ \\
\hline Spontaneous & 10,295 & 19.7 & 8,865 & 16.5 & $0.80^{*}$ & $0.75-0.87$ \\
\hline PROM & 5,410 & 17.7 & 4,513 & 16.8 & 0.94 & $0.85-1.05$ \\
\hline 28-31 wks: latrogenic & 17,001 & 4.88 & 22,023 & 4.29 & $0.87^{*}$ & $0.79-0.96$ \\
\hline Spontaneous & 29,996 & 3.66 & 16,913 & 3.15 & $0.86^{*}$ & $0.76-0.96$ \\
\hline PROM & 9,181 & 3.67 & 7,351 & 3.14 & 0.85 & $0.72-1.01$ \\
\hline 32-33 wks: latrogenic & 17,938 & 2.55 & 25,068 & 2.96 & $0.76^{*}$ & $0.67-0.87$ \\
\hline Spontaneous & 26,093 & 1.62 & 23,136 & 1.41 & 0.87 & $0.75-1.01$ \\
\hline PROM & 11,292 & 1.30 & 9,075 & 1.27 & 0.97 & $0.76-1.24$ \\
\hline 34-36 wks: latrogenic & 101,903 & 0.88 & 168,239 & 0.67 & $0.75^{*}$ & $0.69-0.82$ \\
\hline Spontaneous & 206,090 & 0.48 & 205,518 & 0.36 & $0.75^{*}$ & $0.68-0.82$ \\
\hline PROM & 40,205 & 0.55 & 31,688 & 0.47 & 0.85 & $0.69-1.05$ \\
\hline 24-36 wks: latrogenic & 144,422 & 2.48 & 225,532 & 1.86 & $0.75^{*}$ & $0.71-0.78$ \\
\hline Spontaneous & 262,474 & 1.56 & 254,432 & 1.22 & $0.78^{*}$ & $0.74-0.81$ \\
\hline PROM & 66,088 & 2.51 & 52,627 & 2.38 & 0.95 & $0.88-1.02$ \\
\hline \multicolumn{7}{|l|}{ Twins } \\
\hline 24-27 wks: latrogenic & 1,953 & 20.1 & 3,305 & 17.7 & $0.86^{*}$ & $0.74-0.99$ \\
\hline Spontaneous & 1,955 & 25.3 & 1,685 & 19.8 & $0.73^{*}$ & $0.62-0.85$ \\
\hline PROM & 1,035 & 24.3 & 1,132 & 18.7 & $0.72^{*}$ & $0.59-0.88$ \\
\hline 28-31 wks: latrogenic & 4,607 & 3.30 & 8,144 & 2.74 & 0.83 & $0.67-1.02$ \\
\hline Spontaneous & 3,848 & 2.86 & 4,060 & 2.14 & $0.74^{*}$ & $0.56-0.99$ \\
\hline PROM & 2,149 & 2.75 & 2,333 & 2.53 & 0.92 & $0.64-1.32$ \\
\hline 32-33 wks: latrogenic & 6,290 & 1.95 & 12,076 & 0.64 & $0.67^{*}$ & $0.47-0.94$ \\
\hline Spontaneous & 5,799 & 0.47 & 5,798 & 0.50 & 1.07 & $0.64-1.82$ \\
\hline PROM & 2,195 & 0.68 & 2,575 & 0.78 & 1.14 & $0.58-2.23$ \\
\hline 34-36 wks: latrogenic & 29,476 & 0.42 & 57,447 & 0.26 & $0.63^{*}$ & $0.50-0.80$ \\
\hline Spontaneous & 24,862 & 0.32 & 23,054 & 0.20 & $0.64^{*}$ & $0.45-0.92$ \\
\hline PROM & 3,768 & 0.58 & 4,180 & 0.31 & 0.53 & $0.27-1.06$ \\
\hline 24-36 wks: latrogenic & 42,326 & 1.72 & 80,972 & 1.28 & $0.74^{*}$ & $0.67-0.82$ \\
\hline Spontaneous & 36,464 & 1.95 & 34,597 & 1.43 & $0.73^{*}$ & $0.66-0.82$ \\
\hline PROM & 9,147 & 3.79 & 10,220 & 2.97 & $0.78^{*}$ & $0.66-0.91$ \\
\hline
\end{tabular}

${ }^{*}$ p-value $<0.05$ 
Table 3 Temporal Changes in Neonatal Mortality or Serious Neonatal Morbidity† by Plurality, Gestational Age and Subtype of Preterm Birth, United States, 1995-96 and 2004-05

\begin{tabular}{|c|c|c|c|c|c|c|}
\hline \multirow[b]{2}{*}{$\begin{array}{l}\text { Plurality and gestational age and } \\
\text { preterm birth subtype }\end{array}$} & \multicolumn{2}{|r|}{$1995-96$} & \multicolumn{2}{|r|}{ 2004-05 } & \multirow[b]{2}{*}{$\begin{array}{l}\text { Odds ratio (2004-05 } \\
\text { vs 1995-96) }\end{array}$} & \multirow[b]{2}{*}{$\begin{array}{l}95 \% \\
\text { confidence } \\
\text { intervals }\end{array}$} \\
\hline & $\begin{array}{l}\text { Live } \\
\text { births }\end{array}$ & $\begin{array}{l}\text { Neonatal outcomest/ } \\
100 \text { live births }\end{array}$ & $\begin{array}{l}\text { Live } \\
\text { births }\end{array}$ & $\begin{array}{l}\text { Neonatal outcomest/ } \\
100 \text { live births }\end{array}$ & & \\
\hline \multicolumn{7}{|l|}{ Singletons } \\
\hline 24-27 wks: latrogenic & 6,418 & 49.0 & 7,532 & 42.9 & $0.78^{*}$ & $0.73-0.83$ \\
\hline Spontaneous & 8,569 & 52.1 & 6,683 & 45.5 & $0.77^{*}$ & $0.72-0.82$ \\
\hline PROM & 4,728 & 51.0 & 3,338 & 49.9 & 0.95 & $0.87-1.04$ \\
\hline 28-31 wks: latrogenic & 14,329 & 28.4 & 16,008 & 23.7 & $0.78^{*}$ & $0.74-0.82$ \\
\hline Spontaneous & 16,486 & 23.1 & 12,501 & 20.5 & $0.86^{*}$ & $0.82-0.91$ \\
\hline PROM & 8,083 & 26.9 & 5,402 & 26.3 & 0.97 & $0.89-1.04$ \\
\hline 32-33 wks: latrogenic & 14,973 & 15.8 & 18,051 & 13.3 & $0.82^{*}$ & $0.77-0.87$ \\
\hline Spontaneous & 21,469 & 10.7 & 17,124 & 9.50 & $0.88^{*}$ & $0.82-0.94$ \\
\hline PROM & 9,955 & 12.3 & 6,662 & 12.9 & 1.06 & $0.97-1.16$ \\
\hline 34-36 wks: latrogenic & 86,586 & 5.69 & 119,807 & 4.49 & $0.78^{*}$ & $0.75-0.81$ \\
\hline Spontaneous & 173,286 & 2.73 & 152,483 & 2.43 & $0.89^{*}$ & $0.85-0.93$ \\
\hline PROM & 35,844 & 4.83 & 22,801 & 5.64 & $1.18^{*}$ & $1.09-1.27$ \\
\hline 24-36 wks: latrogenic & 122,306 & 11.9 & 161,398 & 9.17 & $0.75^{*}$ & $0.73-0.77$ \\
\hline Spontaneous & 219,810 & 6.95 & 188,791 & 5.80 & $0.82^{*}$ & $0.80-0.84$ \\
\hline PROM & 58,610 & 12.9 & 38,203 & 13.7 & $1.07^{*}$ & 1.03-1.12 \\
\hline \multicolumn{7}{|l|}{ Twins } \\
\hline 24-27 wks: latrogenic & 1,640 & 49.1 & 2,477 & 42.0 & $0.83^{*}$ & $0.73-0.94$ \\
\hline Spontaneous & 1,671 & 64.1 & 1,303 & 51.7 & $0.69^{*}$ & $0.60-0.80$ \\
\hline PROM & 933 & 59.3 & 844 & 55.2 & 0.85 & $0.70-1.02$ \\
\hline 28-31 wks: latrogenic & 3,875 & 25.2 & 5,967 & 21.6 & $0.86^{*}$ & $0.78-0.94$ \\
\hline Spontaneous & 3,312 & 26.7 & 3,205 & 23.7 & $0.85^{*}$ & $0.76-0.95$ \\
\hline PROM & 1,889 & 30.9 & 1,679 & 28.5 & 0.89 & $0.77-1.03$ \\
\hline 32-33 wks: latrogenic & 5,252 & 12.0 & 8,930 & 11.4 & 0.95 & $0.85-1.06$ \\
\hline Spontaneous & 4,981 & 10.8 & 4,540 & 10.9 & 1.02 & $0.90-1.16$ \\
\hline PROM & 1,986 & 14.7 & 1,839 & 13.7 & 0.92 & $0.77-1.10$ \\
\hline 34-36 wks: latrogenic & 24,983 & 4.25 & 42,236 & 3.65 & $0.84^{*}$ & $0.78-0.91$ \\
\hline Spontaneous & 21,432 & 3.52 & 18,322 & 3.47 & 1.03 & $0.93-1.14$ \\
\hline PROM & 3,372 & 5.60 & 2,975 & 6.62 & 1.19 & $0.97-1.47$ \\
\hline 24-36 wks: latrogenic & 35,750 & 9.72 & 59,610 & 8.20 & $0.84^{*}$ & $0.81-0.88$ \\
\hline Spontaneous & 31,396 & 10.3 & 27,370 & 9.36 & $0.92^{*}$ & 0.87-0.97 \\
\hline PROM & 8,180 & 19.8 & 7,337 & 19.0 & 0.95 & $0.88-1.03$ \\
\hline
\end{tabular}

†Neonatal mortality or serious neonatal morbidity included (neonatal death, 5 -minute Apgar score $\leq 3$, neonatal seizures or assisted ventilation for $\geq 30$ minutes). *p-value $<0.05$

Births with missing information about serious neonatal morbidity were excluded. Births recorded on new birth certificates in 2004-05 were excluded due to a different definition of assisted ventilation.

the temporal changes in the 2 other subtypes of preterm birth $(P$ value $<0.001$ for both contrasts, Table 3$)$. The reductions in neonatal mortality/serious neonatal morbidity in the iatrogenic and spontaneous preterm birth groups were observed at early preterm gestation as well as at late preterm gestation; among live births following preterm PROM, the temporal increase in neonatal mortality/morbidity was observed in the 34-36 week group (Table 3).

Among twins, neonatal mortality/serious neonatal morbidity rates declined significantly following iatrogenic preterm birth and spontaneous preterm birth, while neonatal mortality/serious neonatal morbidity rates among preterm births following PROM did not change significantly (Table 3 ). The neonatal mortality/serious neonatal morbidity decrease among iatrogenic preterm births was larger in magnitude than that among spontaneous preterm births (odds ratios $=0.84,95 \%$ CI:0.81-0.88 vs. $0.92,95 \%$ CI:0.87-0.97; $P$ value for difference in odds ratios $=0.01$ ). Significant reductions in neonatal mortality/morbidity among twin live births following iatrogenic preterm birth were observed at 24-27, 28-31 and 34-36 weeks but not 
among those born at 32-33 weeks. Among twins born following spontaneous preterm birth, neonatal mortality/serious neonatal morbidity was significantly reduced at 24-27 and 28-31 weeks but not at 32-33 and 34-36 weeks, while among twins born following PROM, neonatal mortality/ morbidity was not significantly reduced in any gestational age group (Table 3 ).

Supplementary analyses showed that trends in neonatal mortality/serious neonatal morbidity remained unchanged even after the exclusion of infants with congenital anomalies from the analysis. There was a significantly larger temporal decline in neonatal mortality/ serious neonatal morbidity following iatrogenic preterm birth (odds ratio $=0.80,95 \% \mathrm{CI}: 0.78-0.82$ ) than following spontaneous preterm birth (odds ratio $=0.92,95 \%$ CI:0.90-0.95; $P$ value for difference in odds ratios $<0.001)$. Similar results were also obtained when analysis was restricted to the US states that did not introduce the new birth certificate form in 2004 or 2005. Preterm birth rates were similar between states that did and did not introduce a new birth certificate (10.7 and 10.5 per 100 live births, respectively). Supplementary analyses carried out among older mothers ( $\geq 35$ years) showed that between 1995-96 and 2004-05, neonatal mortality/ serious neonatal morbidity among singletons born preterm following iatrogenic delivery, declined from 10.3 to 7.4 per 100 live births, odds ratio $=0.69$ (95\%CI: 0.64 $0.75)$; among preterm infants born spontaneously the composite outcome increased from 6.5 to 7.1 per 100 live births, odds ratio $=1.10$ (95\%CI: 1.03-1.18). Neonatal mortality/serious neonatal morbidity rates remained relatively stable among infants born following PROM (12.7 and 13.2 per 100 live births), odds ratio $=1.05$ (95\%CI: 0.95-1.16).

\section{Discussion}

We have shown that the temporal increase in preterm birth in the United States between 1995 and 2005 was primarily due to an increase in iatrogenic preterm birth at late preterm gestation among both singletons and twins. This increase in medically indicated preterm birth coincided with reductions in stillbirth rates and neonatal mortality rates. Also, infants born following medically indicated preterm birth showed larger reductions in neonatal mortality and serious neonatal morbidity rates when compared with infants born following spontaneous preterm birth. Neonatal mortality/serious neonatal morbidity rates among infants born following preterm PROM showed a temporal increase among singletons and no significant change among twins.

Our study and previous studies [6,13,14] show that medically indicated preterm birth is the primary cause of the recent increase in preterm birth. This is particularly evident among twins, among whom increases in medically indicated preterm birth have resulted in declines in spontaneous preterm birth. Nevertheless, the various factors responsible for the overall increases in preterm birth (e.g., obstetric intervention, older maternal age and multi-fetal pregnancy) are not mutually exclusive. For instance, population increases in older maternal age lead to increases in medically indicated preterm birth because older maternal age is a risk factor for fetal growth restriction, perinatal mortality and serious neonatal morbidity [26-28].

The reasons for the observed differences in neonatal mortality and serious neonatal morbidity reductions observed among the iatrogenic and spontaneous preterm birth groups probably relate to changes in obstetric surveillance and management. High risk pregnancies with suspected fetal compromise are more carefully monitored currently, with early delivery intervention if the benefits of delivery are deemed to outweigh the risks of preterm birth and expectant management. Given the temporal advances in neonatal care, this effect would be expected mainly at late preterm gestation, when the preterm birth poses less risk to the newborn as compared to earlier gestation. Correspondingly, our findings showed the largest decline in neonatal mortality/serious neonatal morbidity among iatrogenic preterm births which occurred at late preterm gestation. Closer fetal surveillance may also improve outcomes by ensuring that prophylactic antenatal corticosteroid therapy is used, unlike in cases of spontaneous preterm birth where the unexpected onset of labour may preclude such prophylaxis. Maternal transport to a higher level perinatal care facility for labour induction or caesarean delivery may have also contributed to the temporal improvement in neonatal outcomes following iatrogenic delivery. In addition, iatrogenic preterm birth may be carried out for less severe indications in recent years as compared with past years, as the improvements in neonatal care allow for intact survival of preterm infants, especially at late preterm gestation. Although audits of indications for preterm birth show that medically indicated preterm birth is mostly unavoidable and carried out typically for severe or unstable medical/obstetric conditions such as severe preeclampsia or fetal compromise, a small proportion of iatrogenic preterm births may be without a clear medical indication $[29,30]$. Finally, increases in prenatal diagnosis and pregnancy termination during the study period may have contributed to the differences in trends in neonatal mortality/ serious neonatal morbidity following spontaneous and iatrogenic delivery. However, the differences in temporal trends in neonatal mortality/serious neonatal morbidity by preterm birth subtype persisted even after exclusion of infants with congenital anomalies, rendering this explanation unlikely. 
The lack of a temporal improvement in neonatal mortality and in neonatal mortality/serious neonatal morbidity among infants born after preterm premature rupture of membranes over 12 hours is concerning. Such infants constitute approximately $1 \%$ of singleton live births and approximately $5 \%$ of twin live births. Neonatal mortality rates in this preterm category are currently high and the absence of a temporal decline in neonatal mortality/serious neonatal morbidity suggests that this subgroup has not benefitted from recent improvements in obstetric and neonatal care. Research needs to be directed at improving management options for this condition.

The differential temporal reductions in neonatal mortality/serious neonatal morbidity among the iatrogenic and spontaneous preterm subgroups were not evident in contrasts of neonatal mortality. Although reductions in neonatal mortality were somewhat larger among infants born after iatrogenic preterm birth compared with those born following spontaneous preterm birth (odds ratio 0.75 vs. 0.78 ), this difference was not statistically significant. One possible reason for this may be the lesser frequency of neonatal mortality i.e., the lack of a significant difference could have arisen due to a lesser study power.

The limitations of our study include a potential misclassification of preterm birth subtypes. Some cases of spontaneous preterm labour or PROM who were delivered by caesarean for indications such as fetal compromise may have been misclassified as iatrogenic in our study. This problem arose because our data source did not include details regarding the onset of labour. However, the misclassification introduced because of this is likely small as studies from other more clinically focussed databases (which include information on labour onset) have shown similar proportions of iatrogenic and spontaneous preterm births. For instance, a study from British Columbia, Canada [30], showed that $43 \%$ of preterm births in 2005 occurred following preterm labour induction or cesarean delivery in the absence of labour (compared with $42 \%$ in 2004-05 in this study). The categorization of preterm birth has been the source of some debate in the past $[31,32]$. Although each subtype of preterm birth may have a different implication for preventive efforts [33], etiologic pathways are complex and in many instances overlap $[31,34]$.

Another limitation arises because we were not able to utilize data on neonatal morbidity from those states that introduced the new birth certificate forms in 2004 and 2005 due to the incompatibility of definitions for assisted ventilation. However, sensitivity analyses showed that this had a minor impact on our findings. In addition, newborns from states that introduced the new birth certificate during 2004-05 had similar characteristics as compared with infants born in the other states (data not shown). Disease specific information, such as occurrence of intraventricular hemorrhage, necrotizing entrerocolitis or respiratory distress syndrome, was not available in the US data. Instead, we used a composite outcome including neonatal mortality or severe neonatal morbidity, the latter being approximated by Apgar score at 5 minutes $<=3$, prolonged ventilation, and neonatal seizures. This composite outcome was chosen to identify neonates who died or those at a high risk of infant death or disability. This composite outcome has been used in previous studies [35] and is strongly associated with adverse outcomes in long-term follow-up studies [36,37]. Data from California, which did not report the clinical estimate of gestation, was excluded from our study (13\% of births). This represents a limitation of our study but is balanced by the use of an accurate estimate of gestational age.

\section{Conclusion}

In summary, our study shows that recent increases in obstetric intervention in the United States have resulted in larger declines in rates of neonatal mortality and serious neonatal morbidity among infants born following iatrogenic preterm birth as compared with infants born following spontaneous preterm birth. On the other hand, neonatal mortality/serious neonatal morbidity rates among infants born following PROM showed a temporal increase among singletons and no significant change among twins. Whereas our findings on iatrogenic preterm birth are encouraging, they highlight the need for improving outcomes among preterm infants born following preterm premature rupture of membranes. More research is needed to identify the underlying maternal and fetal conditions that lead to preterm delivery in order to develop targeted interventions to prevent adverse neonatal outcomes resulting from preterm birth.

\section{Abbreviations \\ OR: odds ratio; Cl: confidence interval; PROM: premature rupture of membranes; NCHS: National Centre for Health Statistics.}

\section{Acknowledgements}

$\mathrm{SL}$ is supported by a post-doctoral fellowship award from the Michael Smith Foundation for Health Research (MSFHR), JAH is supported by post-doctoral fellowship awards from the Canadian Institutes of Health Research and the MSFHR and KSJ's work is supported by the Child and Family Research Institute.

\section{Author details}

'Department of Obstetrics \& Gynaecology, University of British Columbia and the Women's Hospital and Health Centre of British Columbia, Vancouver, Canada. ${ }^{2}$ School of Population and Public Health, University of British Columbia, Vancouver, Canada.

\section{Authors' contributions}

All authors contributed to the conception and design of the study and in the preparation of the manuscript. All authors read and approved the final manuscript. 


\section{Competing interests}

The authors declare that they have no competing interests.

Received: 24 February 2011 Accepted: 25 May 2011

Published: 25 May 2011

\section{References}

1. Hamilton BE, Martin JA, Ventura SJ: Births: Preliminary data for 2007. National vital statistics reports. Hyattsville, MD: National Center for Health Statistics; 2009:57:no. 12

2. Martin JA, Kirmeyer S, Osterman M, Shepherd RA: Born a Bit Too Early: Recent Trends in Late Preterm Births. NCHS Data Brief 2009, 24:1-8.

3. Health Canada: Canadian Perinatal Health Report, 2000. Ottawa: Minister of Public Works and Government Services Canada, 2000; 2000.

4. Births 2007. Statistics Canada. Minister of Industry. Ottawa; 2009, Catalogue No. 84F0210X

5. Joseph KS, Demissie K, Kramer MS: Trends in obstetric intervention, stillbirth and preterm birth. Semin Perinatol 2002, 26:250-9.

6. Ananth CV, Joseph KS, Oyelese Y, Demissie K, Vintzileos AM: Trends in preterm birth and perinatal mortality among singletons: United States, 1989 through 2000. Obstet Gynecol 2005, 105(5):1084-91.

7. Tough SC, Newburn-Cook C, Johnston DW, Svenson LW, Rose S, Belik J: Delayed childbearing and its impact on population rate changes in lower birth weight, multiple birth, and preterm delivery. Pediatrics 2002, 109(3):399-403.

8. Martin JA, Hamilton BE, Sutton PD, Ventura SJ: Births; Final data for 2006. In National Vital Statistics Reports. Volume 57. Hyattsville, MD: National Centre for Health Statistics; 2009, Report No: 7.

9. Fell DB, Joseph KS, Dodds L, Allen AC, Jangaard K, Van den HM: Changes in maternal characteristics in Nova Scotia, Canada from 1988 to 2001. Can J Public Health 2005, 96(3):234-8.

10. Health Canada: Canadian Perinatal Health Report, 2008 Edition. Ottawa:2008, Catalogue No HP10-12/2008E.

11. Blondel B, Kaminski M: Trends in the occurrence, determinants, and consequences of multiple births. Semin Perinatol 2002, 26(4):239-49.

12. Blickstein I, Keith LG: Aging, twinning, and perinatal outcomes. Fertil Steril 2003, 79(3):661-2.

13. Barros FC, Velez MP: Temporal trends of preterm birth subtypes and neonatal outcomes. Obstet Gynecol 2006, 107(5):1035-41.

14. Norman JE, Morris C, Chalmers J: The effect of changing patterns of obstetric care in Scotland (1980-2004) on rates of preterm birth and its neonatal consequences: perinatal database study. PLoS Med 2009, 6(9): e1000153

15. Chen A, Feresu SA, Barsoom MJ: Heterogeneity of preterm birth subtypes in relation to neonatal death. Obstet Gynecol 2009, 114(3):516-22.

16. Department of Health and Human Services, Centre for Disease Control and Prevention, National Centre for Health Statistics, Division of Vital Statistics: Public Use Data File Documentation, 1995 Period Linked Birth/Infant Death Data Set.[http://www.cdc.gov/nchs/data_access/Vitalstatsonline.htm], Accessed April 2011.

17. Department of Health and Human Services, Centre for Disease Control and Prevention, National Centre for Health Statistics, Division of Vital Statistics: Public Use Data File Documentation, 2005 Period Linked Birth/Infant Death Data Set.[http://www.cdc.gov/nchs/data_access/Nitalstatsonline.htm], Accessed April 2011

18. Wier ML, Pearl M, Kharrazi M: Gestational age estimation on United States livebirth certificates: a historical overview. Paediatr Perinat Epidemiol 2007, 21(Suppl 2):4-12

19. Joseph KS, Huang L, Liu S, Ananth CV, Allen AC, Sauve R, et al: Reconciling the high rates of preterm and postterm birth in the United States. Obstet Gynecol 2007, 109(4):813-22.

20. Mustafa G, David RJ: Comparative accuracy of clinical estimate versus menstrual gestational age in computerized birth certificates. Public Health Rep 2001, 116(1):15-21.

21. Ananth CV: Menstrual versus clinical estimate of gestational age dating in the United States: temporal trends and variability in indices of perinatal outcomes. Paediatr Perinat Epidemiol 2007, 21(Suppl 2):22-30.

22. Sepkowitz S: International rankings of infant mortality and the United States' vital statistics natality data collecting system-failure and success. Int J Epidemiol 1995, 24(3):583-8.
23. Sachs BP, Fretts RC, Gardner R, Hellerstein S, Wampler NS, Wise PH: The impact of extreme prematurity and congenital anomalies on the interpretation of international comparisons of infant mortality. Obstet Gynecol 1995, 85(6):941-6.

24. Kramer MS, Platt RW, Yang H, Haglund B, Cnattingius S, Bergsjo P. Registration artifacts in international comparisons of infant mortality. Paediatr Perinat Epidemiol 2002, 16(1):16-22.

25. Breslow NE, Day NE: Statistical methods in cancer research: Vol. I - The analysis of case-control studies. Lyon: IARC; 1980, Scientific Publ. No 32.

26. Joseph KS, Allen AC, Dodds L, Turner LA, Scott $H$, Liston R: The perinatal effects of delayed childbearing. Obstet Gynecol 2005, 105:1410-1418.

27. Aldous $M B$, Edmonson MB: Maternal age at first childbirth and risk of low birth weight and preterm delivery in Washington State. JAMA 1993, 270:2574-2577.

28. Jolly M, Sebire N, Harris J, Robinson S, Regan L: The risks associated with pregnancy in women aged 35 years or older. Hum Reprod 2000, 15:2433-2437.

29. Holland MG, Refuerzo JS, Ramin SM, Saade GR, Blackwell SC: Late preterm birth: how often is it avoidable? Am J Obstet Gynecol 2009, 201(4):404.

30. Joseph KS, Barker S, Dale S, Lee L, McMaster R, Miyazaki L: Preterm birth in British Columbia. British Columbia Perinatal Health Program. Vancouver: 2008 [http://www.perinatalservicesbc.ca//sites/bcrcp/files/Publications/ BCPHP_PretermBirthlnBC.pdf], Accessed May 2011.

31. Savitz DA, Dole N, Herring AH, Kaczor D, Murphy J, Siega-Riz AM, et al: Should spontaneous and medically indicated preterm births be separated for studying aetiology? Peadiatric and Perinatal Epidemiology 2005, 19:97-105.

32. Berkowitz GS, Blackmore-Prince C, Lapinski RH, Savitz DA: Risk factors for preterm birth subtypes. Epidemiology 1998, 9:279-285.

33. Joseph KS, Theory of obstetrics: An epidemiologic framework for justifying medically indicated early delivery. BMC Pregnancy and Childbirth 2007, 7:4.

34. Ananth CV, Vintzileos AM: Epidemiology of preterm birth and its clinical subtypes. J Maternal-Fetal and Neonatal Medicine 2006, 199(12):773-782.

35. Joseph KS, Fahey J, Platt RW, Liston RM, Lee SK, Sauve R, Liu S, Allen AC, Kramer M: An outcome-based approach for the creation of fetal growth standards: Do singletons and twins need separate standards? Am J Epid 2009, 169(5):616-624.

36. Mellits $E D$, Holden KR, Freeman JM: Neonatal seizures. II. A multivariate analysis of factors associated with outcome. Pediatrics 1982, 70(2):177-185

37. Eilenberg $\mathrm{JH}$, Nelson KB: Cluster of perinatal events identifying infants at high risk for death or disability. J Pediatr 1988, 113(3):546-552

\section{Pre-publication history}

The pre-publication history for this paper can be accessed here: http://www.biomedcentral.com/1471-2393/11/39/prepub

doi:10.1186/1471-2393-11-39

Cite this article as: Lisonkova et al:: Temporal trends in neonata outcomes following iatrogenic preterm delivery. BMC Pregnancy and Childbirth 2011 11:39.

\section{Submit your next manuscript to BioMed Central and take full advantage of:}

- Convenient online submission

- Thorough peer review

- No space constraints or color figure charges

- Immediate publication on acceptance

- Inclusion in PubMed, CAS, Scopus and Google Scholar

- Research which is freely available for redistribution 